\title{
Erratum
}

Nephrologe 2015 · 10:252-252

DOI 10.1007/s11560-015-1002-5

Online publiziert: 15. Mai 2015

c) Springer-Verlag Berlin Heidelberg 2015

\section{Czock ${ }^{1} \cdot$ F. Keller ${ }^{2}$}

${ }^{1}$ Klinische Pharmakologie und Pharmakoepidemiologie, Universitätsklinikum Heidelberg, Krehl-Klinik, Heidelberg

${ }^{2}$ Nephrologie, Universitätsklinikum Ulm, Ulm

\section{Erratum zu:}

\section{Antibiotikadosierung bei Dialysepatienten}

Im Beitrag muss es in der Tabelle 1 bei der Dosierung von Trimethoprim/Sulfamethoxazol bei normaler Nierenfunktion , $5 / 25 \mathrm{mg} / \mathrm{kg}^{\text {“ }}$ anstelle von „5/25 mg/ $\mathrm{kg} / \mathrm{Tag}$ " heißen.

Bei der Dosierung von Cefotaxim wurde nur die höchste denkbare Dosis für schwerstkranke Patienten aufgenommen. Die Empfehlung für Cefotaxim sollte, wie nachfolgend in Tabelle 1 aufgeführt, lauten:

\section{Korrespondenzadresse}

PD Dr. D. Czock

Klinische Pharmakologie und Pharmakoepidemiologie,

Universitätsklinikum Heidelberg, Krehl-Klinik Im Neuenheimer Feld 410, 69120 Heidelberg david.czock@med.uni-heidelberg.de

Die Online-Version des Originalartikels ist unter doi: 10.1007/s11560-014-0928-3 zu finden.

\begin{tabular}{|c|c|c|c|c|c|c|c|c|c|}
\hline \multirow[t]{3}{*}{ Arzneimittel } & & \multicolumn{3}{|c|}{ Normale Nierenfunktion } & \multicolumn{4}{|l|}{ Dialysepatienten } & \multirow[t]{3}{*}{ Kommentare } \\
\hline & & \multirow[t]{2}{*}{ Startdosis } & \multicolumn{2}{|c|}{ Erhaltungsdosierung } & \multirow[t]{2}{*}{ Startdosis } & \multicolumn{3}{|c|}{ Erhaltungsdosierung } & \\
\hline & & & Dosis & Intervall & & Dosis & Intervall & Ersatzdosis & \\
\hline Cefotaxim & iv. & & $\begin{array}{l}2000 \\
(3000) \mathrm{mg}\end{array}$ & $(6-) 8 \mathrm{~h}$ & $2000(3000) \mathrm{mg}$ & $1000(1500) \mathrm{mg}$ & $(6-) 12 \mathrm{~h}$ & & $\begin{array}{l}\text { Am Dialysetag } \\
\text { nach HD }\end{array}$ \\
\hline
\end{tabular}

